Document downloaded from:

http://hdl.handle.net/10251/44811

This paper must be cited as:

García Gil, PJ.; Albertos Pérez, P. (2013). Robust tuning of a generalized predictor-based controller for integrating and unstable systems with long time-delay. Journal of Process Control. 23(8):1205-1216. doi:10.1016/j.jprocont.2013.07.008.

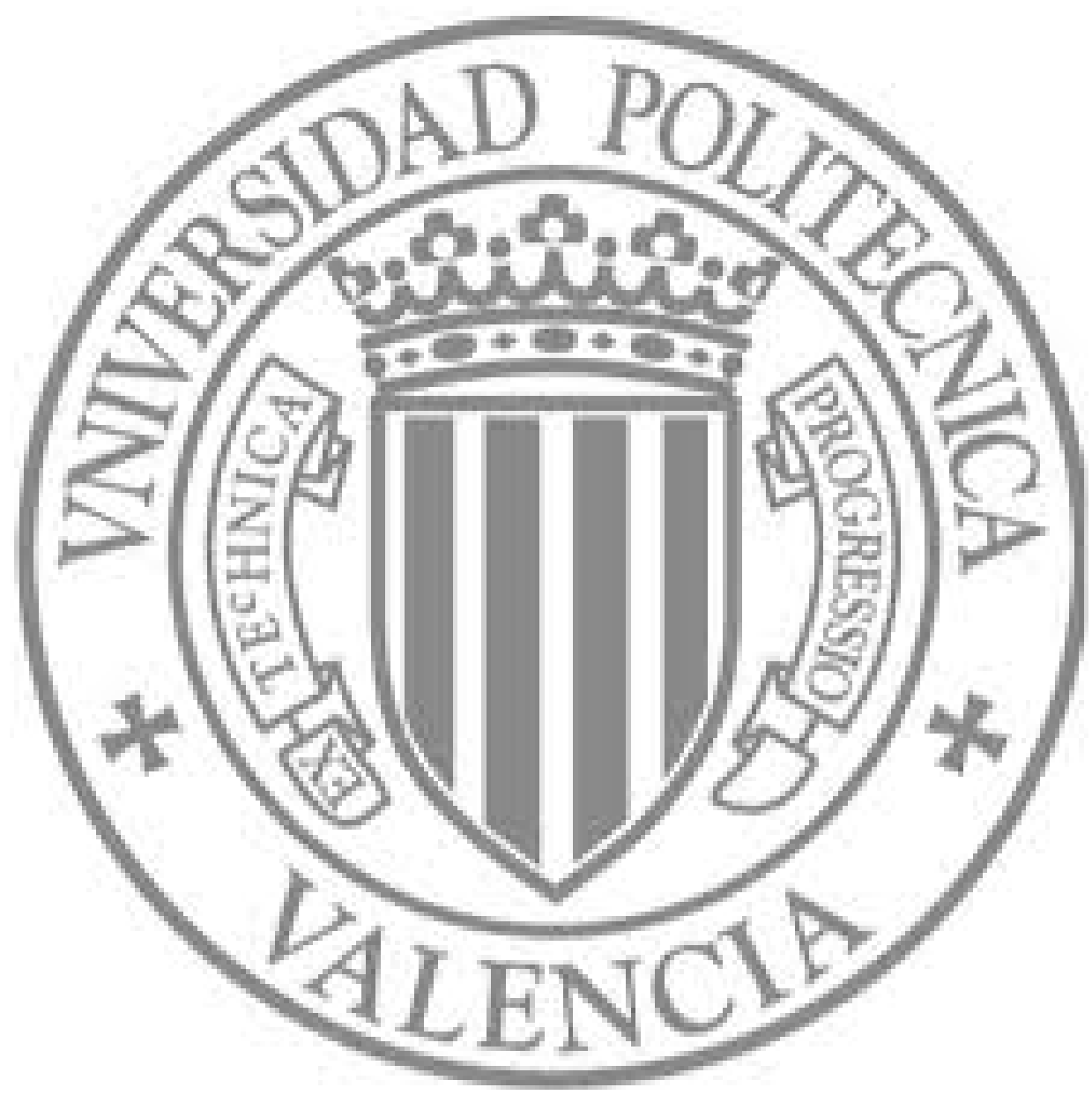

The final publication is available at

http://dx.doi.org/10.1016/j.jprocont.2013.07.008

Copyright Elsevier 


\title{
Robust tuning of a Generalized Predictor-based controller for integrating and unstable systems with long time-delay
}

\author{
Pedro García, Pedro Albertos* \\ Department of Systems Engineering and Control, Instituto de Automática e Informática Industrial \\ Universitat Politècnica de València, Valencia, Spain
}

\begin{abstract}
In this work, a general structure to control long time-delay plants is proposed and an easy methodology to tune the control parameters is outlined. All the sensitivity transfer functions are delay free. The proposed scheme is equivalent to the Smith Predictor but able to cope with any kind of systems, including non-minimum phase, unstable and integrating plants. The controllers are designed based on the delay free model. Contrary to other approaches, other than for the digital implementation, no delay approximation is used. A tuning parameter is provided in order to reach an intuitive tradeoff between performance and robust stability. A comparative analysis with respect to recently successful proposals shows a substantial improvement in the performance/robustness tradeoff as well as in the tuning process.
\end{abstract}

Keywords: Long time delay, Dead-time compensation, Integrating and unstable systems, Robustness, Smith Predictor.

\section{Introduction}

Most industrial processes are characterized by the presence of time delays [23]. Conventional controllers, such as PID controllers [6], could be used when the dead-time is small but they show poor performance when the process exhibits long dead-times [12]. In these cases, it is convenient to introduce a dead-time compensating (DTC) structure [22].

DTCs structures include a model of the process in order to cope with the dead time. The Smith Predictor (SP) [30] was the first and seminal proposal. As it is well known, the main idea is to generate the undelayed plant response by using the available information: the process input and output and the process model. The main advantage of the SP method is that time delay is eliminated from the characteristic equation (not only from the set-point to output transfer function but from all the sensitivity functions of the closed-loop system). Thus, the analysis and design problem for processes with delay can be translated into a delay-free one. On the other hand, as the SP uses the actual model of the plant, it presents several problems in presence of integrating or unstable processes $[22,11]$, being also sensitive to time delay uncertainty[20].

\footnotetext{
* Corresponding author: Tel.: +34963879570 ; E-mail address: pedro@aii.upv.es (P. Albertos)
}

The control community has devoted a great effort against such drawbacks. Some DTCs have been proposed to simplify and improve the tuning of the controller [14, 13, 12, 32, 21], to reject load disturbances for processes with integration and long dead-time $[5,18,25,35,9,33]$, and to control unstable time-delay systems $[17,31,34,15,16,8,24,1]$. However, for integrating and unstable systems none of the proposed schemas, except those proposed in [24, 1], fulfill the SP principle, the time delay being eliminated from all the sensitivity function of the closed loop system [10].

Several PID-based design methods have been proposed for the control of integrating and unstable processes with time delay. The tuning design can be performed following different approaches (see [27] for a tutorial on the topic) but there is always an approximation in the delay term to obtain the final PID control structure [31]. Some times this PID algorithm is in cascade with a lead-lag first or second order filter [27]. A common drawback in this kind of approaches is that ideal PID controllers are usually proposed, but the tuning of the PID derivative filter is not a simple issue. Small values of the time constant of this filter causes strong control action and poor noise attenuation. On the other hand, large values may drive the system to instability $[6,10]$.

Recently the so called MSP-PID controller has been proposed in [19] to cope with stable, integrating and un- 
stable processes. The proposal can be implemented as a DTC control structure or in a PID form in a two-degree of freedom control structure although a better set-point tracking is obtained in the later implementation form. In this MSP-PID controller, the control parameters of the main controller are obtained by solving an optimization problem, and a second order Padé approximation is used to get an effective realization of the DTC control structure.

In this work, a general structure to control stable, integrating or unstable long delayed plants based on a generalized predictor (GP), [1], is presented and a methodology for an easy and robust tuning of the control parameters is proposed. The control structure includes four blocks: the main controller, an optional set-point filter, the undelayed output predictor and a prediction error filter. A new parameter is introduced in both, the predictor and the prediction error filter to get a tradeoff between performance and robust stability, keeping in mind that the waterbed effect between the sensitivity and complementary sensitivity function is unavoidable [29]. The principal advantages of the proposed structure is the easy tuning and industrial implementation as well as the intuitive effect of the control parameters. The tuning procedure is shown to be simple and easy to apply to a variety of processes, including those with a long deadtime. It is worth to note that, except for the predictor, the remaining blocks are independent of the delay, although the final behavior is unavoidably affected by the delay. A re-tuning of the control structure parameters may reduce this effect.

This paper is organized as follows. First, the ideal, undelayed control situation is reminded. Then, the structure and components of the GP are summarized. The proposed predictor-based control is described and a simple procedure to tune the different parameters in the control structure is outlined. In particular, the use of the new parameter $(\lambda)$ to improve the controlled plant robustness is discussed. Some examples, applying this approach to some typical plants already used in the literature, illustrate the design methodology and the achieved improvements with respect to recently presented alternative approaches. Some comments and suggestions are drafted in the last section.

\section{Problem statement}

An input-output delayed SISO process can be described by

$$
P_{p}(s)=G_{p}(s) e^{-L_{p} s}
$$

where $L_{p}$ is the time-delay and $G_{p}(s)$ represents the undelayed dynamics. Due to modeling errors and uncertainties, the plant model

$$
P(s)=G(s) e^{-L s}
$$

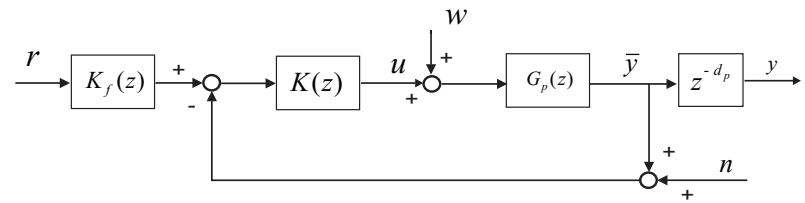

Figure 1. Ideal delay-free loop

differs from the actual plant. Since in any practical application a computer is used to implement the control law [7], it is justified to study the overall closed-loop system in the discrete framework.

Thus, the discrete time (DT) behavior of the actual plant will be represented by the DT transfer function

$$
P_{p}(z)=G_{p}(z) z^{-d_{p}}
$$

where the time delay will be approximated by a multiple of the sampling period ${ }^{1}, T$, being $L_{p}=d_{p} T, d_{p} \in \mathbb{N}$. The assumed plant model is

$$
P(z)=G(z) z^{-d}
$$

For perfect model matching, $P(z)=G(z) z^{-d}=P_{p}(z)$, with $L=L_{p}=d T$.

The ideal setting to design the control is to keep the delay out of the loop and design the control structure for the undelayed plant. Assume a plant as depicted in Figure 1, where the undelayed output $\bar{y}(z)$ is accessible. The ideal system output is

$$
\begin{aligned}
y(z) & =K_{f}(z) \frac{K(z) G(z) z^{-d}}{1+K(z) G(z)} r(z) \\
& +\frac{G(z) z^{-d}}{1+K(z) G(z)} w(z) \\
& -\frac{K(z) G(z) z^{-d}}{1+K(z) G(z)} n(z)
\end{aligned}
$$

The controller $K(z)$ may be designed to achieve some desired performance on the closed-loop disturbance response, whereas the filter $K_{f}(z)$ can be designed to fulfil the tracking performance. If $K(z)$ is properly designed, the system is proved to be internally stable [4].

In general, the undelayed plant output, $\bar{y}$, is not accessible and a model-based predictor should be implemented. The predictor should work for any kind of systems.

\footnotetext{
1 As the control structure should be robust under model parameters uncertainty, the round-off of the fractional delay will not be a problem.
} 


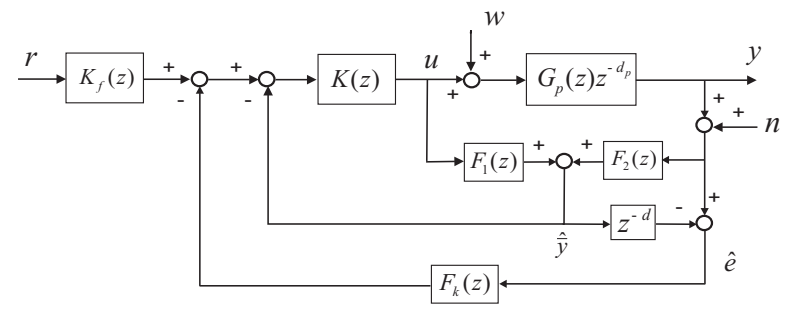

Figure 2. Predictor-based control structure

\section{Generalized Predictor Structure}

In order to design a delay free control, an undelayed output $\bar{y}(z)$ should be predicted based on the current available information: $u(z), y(z)$ and the plant model. The proposed predictor structure is shown in Figure 2.

In this way, the predicted undelayed output will be

$$
\hat{\bar{y}}(z)=F_{1}(z) u(z)+F_{2}(z)[y(z)+n(z)]
$$

In the GP, as initially presented at [1], two stable filters based on the plant model are computed. In this work, in order to improve the robust tuning of the overall closedloop system, a new but equivalent undelayed output predictor $\bar{y}(z)$ is considered. This predictor includes a design parameter, $\lambda$, that can be tuned in order to get an easy and intuitive tradeoff between load disturbance rejection and robust stability.

\subsection{Undelayed output predictor}

Let us consider an equivalent model of the plant (4) represented by

$$
P(z)=G(z) z^{-d}=\frac{N(z)}{D(z)} z^{-d}=\tilde{G}(z, \lambda) \Gamma(z, \lambda) z^{-d}
$$

where the plant zeros are collected in

$$
\Gamma(z, \lambda):=\frac{N(z)}{(z-\lambda)^{m}} ; \quad|\lambda|<1
$$

where $\lambda$ is the tuning parameter and $m$ is the number of zeros on the polynomial $N(z)$.

Remark 1. If $\lambda=0$ and only the non-minimum phase zeros are collected in $N(z)$, the process model decomposition considered in [1] is obtained.

According to (9)-(10), the transfer function $\tilde{G}(z)$ including all the system's poles is

$$
\begin{aligned}
\tilde{G}(z, \lambda) & =\frac{(z-\lambda)^{m}}{D(z)}=\frac{\tilde{N}(z, \lambda)}{D(z)} \\
& =c(z I-A)^{-1} b
\end{aligned}
$$

where $(A, b, c)^{2}$ is a minimum order state space model, very convenient to make easier the following computations.

Let us define also a new transfer function

$$
\tilde{G}^{*}(z, \lambda):=c(z I-A)^{-1} A^{d} b=\frac{\tilde{N}^{*}(z, \lambda)}{D(z)}
$$

Lemma 3.1 For any process, no matter being stable/unstable and minimum/ non-minimum phase, denoting by $(A, b, c)$ a minimum order state space representation of $\tilde{G}(z, \lambda)$, including the possibly unstable part of the transfer function, the stable filters in (8) computed by

$$
\begin{aligned}
& F_{1}(z, \lambda)=\Phi(z, \lambda) \Gamma(z, \lambda) \\
& F_{2}(z, \lambda)=\frac{\tilde{N}^{*}(z, \lambda)}{(z-\lambda)^{m}}
\end{aligned}
$$

where

$$
\Phi(z, \lambda):=c(\lambda) \sum_{i=1}^{d} A^{i-1} b z^{-i}
$$

provide a stable undelayed output estimation.

Proof The proof follows a reasoning similar to that in Lemma 3.1 of [1] (see Remark 1). For the nominal undisturbed plant (9), assuming $w=0$ and $n=0$, the undelayed output is obtained by:

$$
\hat{\bar{y}}(z)=\tilde{G}(z, \lambda) \Gamma(z, \lambda) u(z)
$$

Taking into account (11) and (12), that is ${ }^{3}$

$$
\tilde{G}^{*}=\frac{\tilde{N}^{*}}{(z-\lambda)^{m}} \tilde{G}
$$

adding and subtracting $\tilde{G}^{*} z^{-d}$ from (17) in (16), it can be rewritten as

$$
\hat{\bar{y}}=\left[\tilde{G}-\tilde{G}^{*} z^{-d}+\frac{\tilde{N}^{*}}{(z-\lambda)^{m}} \tilde{G} z^{-d}\right] \Gamma u
$$

But from (14) and (9), it results

$$
\begin{aligned}
F_{2} y & =\frac{\tilde{N}^{*}}{(z-\lambda)^{m}} \tilde{G} \Gamma(z, \lambda) u \\
\hat{\bar{y}} & =\left[\tilde{G}-\tilde{G}^{*} z^{-d}\right] \Gamma u+F_{2} y(z)
\end{aligned}
$$

2 If a controllability canonical representation is chosen, the $\lambda$-parameter only appears in the vector $c(\lambda) \in \Re^{1 x n}$. This is assumed in the following, to simplify the notation.

${ }^{3}$ In the following, the arguments $z$ and $\lambda$, if not needed, are avoided to simplify the notation. 
On the other hand, it is easy to verify that:

$$
\begin{aligned}
(z I-A) \sum_{i=1}^{d} A^{i-1} z^{-i} & =I-A^{d} z^{-d} \\
\sum_{i=1}^{d} A^{i-1} z^{-i} & =(z I-A)^{-1}\left[I-A^{d} z^{d}\right]
\end{aligned}
$$

Thus, premultiplying (18) by $c$ and postmultiplying by $b \Gamma$, it yields $F_{1}$ in the left hand side and the right hand side, taking into account (11) and (12), leads to (13):

$$
F_{1} u=\left(\tilde{G}-\tilde{G}^{*} z^{-d}\right) \Gamma u
$$

proving the lemma.

\subsection{Prediction error compensation}

Due to disturbances and model uncertainties, there is an error in the prediction, such that

$$
\hat{e}=y-\hat{\bar{y}} z^{-d}
$$

This predictor error can be used to improve the behavior of the controlled plant if the control is generated through the predicted undelayed output, as described in the next section.

\section{Control structure}

The predictor-based control structure is shown in Figure 2, where there is a main controller $K(z)$ and the optional set-point filter $K_{f}(z)$. In order to improve the robustness, a predictor error filter $F_{k}(z)$ can be added, being tuned to achieve a tradeoff between performance and robust stability. This filter can be any stable and unitary steady-state gain filter (see $[9,1]$ ).

Lemma 4.1 In the nominal case, from the schema in Figure 2, and according to Lemma 3.1, the following expressions are obtained:

$$
\begin{aligned}
y & =K_{f} \frac{K G z^{-d}}{1+K G} r \\
& +\frac{G z^{-d}}{1+K G} w-\frac{K G z^{-d}}{1+K G} H_{1} w \\
& -\frac{K G z^{-d}}{1+K G} H_{2} n
\end{aligned}
$$

where:

$$
\begin{aligned}
& H_{1}:=\left(F_{1}-F_{k} F_{1} z^{-d}\right) \\
& H_{2}:=\left(F_{2}+F_{k}-F_{k} F_{2} z^{-d}\right)
\end{aligned}
$$

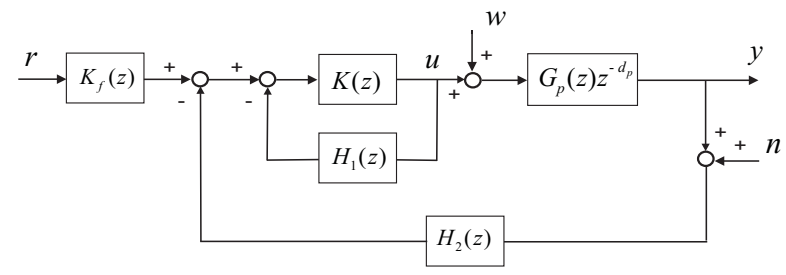

Figure 3. Simplified control structure for analysis proposes.

Proof The control structure depicted in Figure 2 can be simplified as shown in Figure 3, where $H_{1}$ and $H_{2}$ are expressed by (22)-(23).

Thence, the following expressions are obtained:

$$
\begin{aligned}
y & =K_{f} \frac{K P_{p}}{1+K\left(H_{1}+H_{2} P_{p}\right)} r \\
& +\frac{P_{p}}{1+K\left(H_{1}+H_{2} P_{p}\right)} w+\frac{K P_{p} H_{1}}{1+K\left(H_{1}+H_{2} P_{p}\right)} w \\
& +\frac{-K P_{p}}{1+K\left(H_{1}+H_{2} P_{p}\right)} H_{2} n
\end{aligned}
$$

In the nominal case $\left(P_{p}=P\right)$, from Lemma 3.1, it is easy to show that $F_{1}=G-\tilde{G} z^{-d}$, and $F_{2} G z^{-d}=\tilde{G} z^{-d}$, so $F_{1}+F_{2} G z^{-d}=G$.

Thus:

$$
\begin{aligned}
H_{1}+H_{2} P & =F_{1}-F_{k} F_{1} z^{-d}+\left(F_{2}+F_{k}-F_{k} F_{2} z^{-d}\right) P \\
& =F_{1}\left(1-F_{k} z^{-d}\right)+F_{2} P\left(1-F_{k} z^{-d}\right)+F_{k} P \\
& =G
\end{aligned}
$$

Then, replacing (22), (23) and (24) in the above expressions, (19-21) is obtained.

Remark 2. Note that, contrarily to most approaches presented in the literature (except the one proposed in [24]), the characteristic equation of all sensitivity functions is delay-free.

\subsection{Internal stability}

Lemma 4.2 The controlled system depicted in Figure 2, where $K(z)$ has been designed to stabilize $G(z)$, is internally stable.

Proof The proof is straightforward. Note that, in (1921), $K(z)$ has been designed to stabilize $1+K(z) G(z)$, and $H_{1}(z)$ and $H_{2}(z)$ are stable filters.

Remark 3. Note that the design $\lambda$-parameter has no influence in the computation of the predicted undelayed output, and it does not appear in the set-point tracking 
performance. But this parameter can be tuned in order to reach a tradeoff between load disturbance rejection and robust stability in an easy way, as seen in the next section.

\subsection{Tradeoff between performance and robust stability}

In order to evaluate the performance of the proposed undelayed output prediction $F_{k}(z)=1$ is considered in this section.

Performance: A commonly used measurement of disturbance performance is the integral of the absolute error $(I A E)$ index. The lower the IAE index the better the performance is. If the controlled plant is designed to have an over damped closed-loop system, under constant disturbances the $I A E$ is equal to the integral of the error $(I E)$, and an analytical expression can be obtained by (see, for instance, [6])

$$
I A E=\lim _{z \longrightarrow 1} \frac{T}{z-1} G_{y w}(z)
$$

where $T$ is the sampling period and $G_{y w}(z)$ is as described in (20).

Then, the overall IAE is the sum of two terms (20):

$$
\begin{aligned}
I A E & =I A E_{i}+I A E_{G P} \\
& =I A E_{i}+\lim _{z \longrightarrow 1} \frac{T}{z-1}\left(F_{1}-F_{1} z^{-d}\right) \\
& =I A E_{i}+N(1) \frac{\Phi(1, \lambda)}{(1-\lambda)^{m}} d T
\end{aligned}
$$

where $I A E_{i}$ is the contribution of the "ideal" controlled plant (without delay), and the second term is due to the proposed predictor.

The $I A E$ performance can be computed as a function of the parameter $\lambda^{4}$. Then, (26) can be used to compute the value of $\lambda$ to get a desired IAE by using the proposed control scheme. This performance (26) is plotted as a function of $\lambda$ in Figure 4, for the process model of Example 1 in Section 6.

Robustness: For multiplicative uncertainties, that is, if a process transfer function $P_{p}(z)=P(z)(1+W(z))$ is assumed, where $W(z)$ defines the process multiplicative uncertainty bound, the robust stability condition is

\footnotetext{
4 Note that $\Phi(1, \lambda)$ depends on both, $\lambda$ and the process model parameters. In all the examples considered in this work the higher this parameter is the higher the IAE is, but an analytical study of the effect of this parameter for any kind of systems is out of the scope of this work and will be a matter of further research.
}

obtained from the output-noise sensitivity function

$\left\|T_{r} \frac{K G}{1+K G} W\right\|_{\infty}<1$

where

$T_{r}=\left(F_{2} z^{-d}-F_{2}-1\right)=\frac{\tilde{N}^{*}(z) z^{-d}-\tilde{N}^{*}(z)-(z-\lambda)^{m}}{(z-\lambda)^{m}}$

The tuning of $\lambda$ could be done in order to reduce $\left|T_{r}\right|$ in the frequencies of the peak of $\left|\frac{K G}{1+K G} W\right|$, increasing the controlled plant robustness.

But it is well know that for any closed-loop control system the higher the robustness is the lower the performance is obtained, then a tradeoff between both properties should be considered.

The robustness function (28) for the same process model of Example 1 in Section 6 is plotted as a function of $\lambda$ in Figure $4^{5}$. The lower the robustness function the more robust the controlled plant is. This easy and intuitive graphical plot can be used to find some value of $\lambda$ to achieve a tradeoff between IAE and robust stability.

It can be seen that the lower the $\lambda$ parameter is the lower IAE is obtained but there is a considerable increment in the robustness function (28) (i. e., the robustness decreases). Nevertheless, this effect is bounded. Note that if $|\lambda| \geq 1$ the system becomes unstable because an unstable pole is introduced in the predictor scheme. The internal stability is not preserved anymore.

\section{Predictor error filter $F_{k}(z)$ :}

In order to improve the robustness, the predictor error filter $F_{k}(z)$ can be also included. This filter can be any stable and steady-state unitary gain filter, but in order to simplify the number of tuning parameters, in this work, the following filter is considered

$$
F_{k}(z)=\frac{(1-\lambda)^{m}}{(z-\lambda)^{m}}
$$

In that case, the IAE introduced by the DTC structure can be computed by:

$$
I A E_{G P}=\frac{\Phi(1, \lambda) N(1)}{(1-\lambda)^{m}} d T+\frac{\Phi(1, \lambda) N(1)) m T}{(1-\lambda)}
$$

and the robustness function (28) will be $T_{r}=H_{2},(23)$.

\footnotetext{
5 Similar result are obtained for all considered examples in this work.
} 


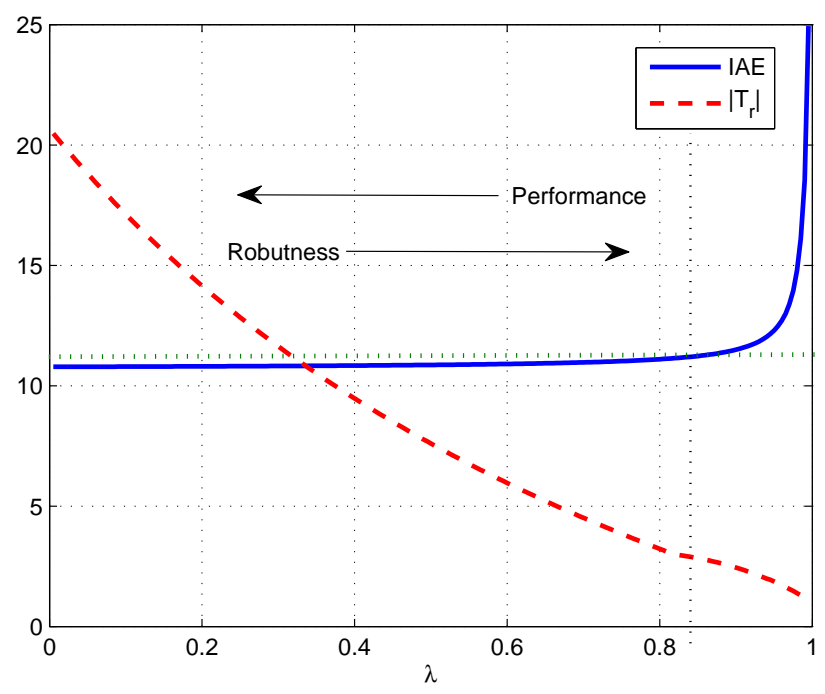

Figure 4. Plot of $I A E$ and $T_{r}$ according to (26) and (28) respectively (Example 1).

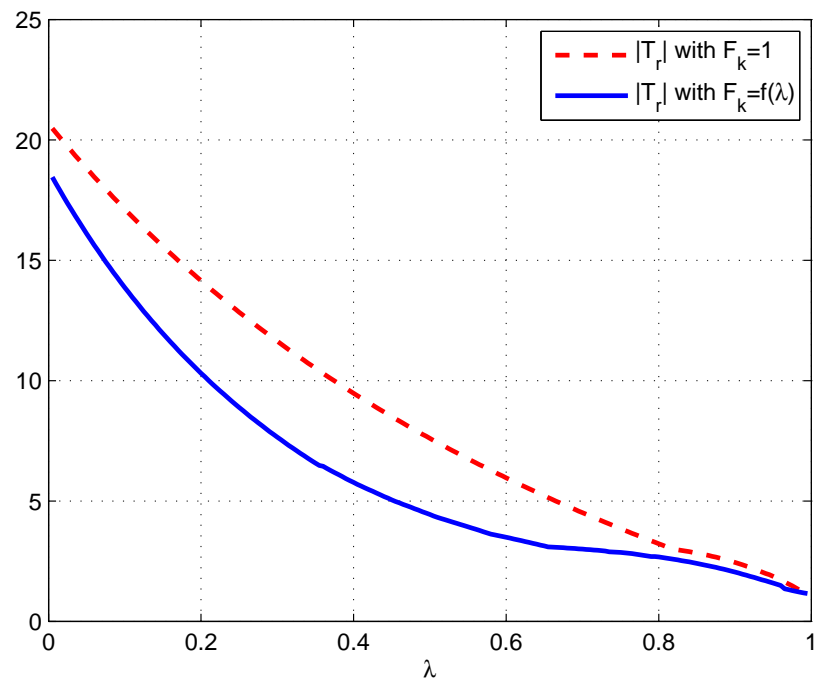

Figure 5. Plot of $T_{r}$ in (28) and (23) respectively.

For the same Example 1 in Section 6, the robustness function (23) is plotted as a function of $\lambda$ in Figure 5, for $F_{k}=1$ (same as in Figure 4) and for $F_{k}(\lambda)(29)$. Note that for the same $\lambda$ the robustness function is lower (the robustness is higher) if the prediction error filter is used. Thus, for a given IAE an increment in the robust stability is obtained, although this effect almost vanishes for $\lambda \longrightarrow 1$.

The tradeoff between performance and robust stability can be tuned in two stages. First, in designing the main controller $K(z)$. Then, by selecting the $\lambda$ filter parameter. The first step is independent of the process time-delay and it can be obtained by any robust design method, leading to an inner control loop weighting these characteristics. Once this step is fulfilled, the global balance between performance and robustness can be fine tuned by the $\lambda$ parameter.

\section{Tuning design procedure}

In this section a general, easy, intuitive and systematic procedure to design the control structure of Figure 2 is outlined. The proposed procedure is as follows:

(1) Design of the main controller $K(z)$ : First, a discrete model of the process is obtained by using an appropriate sampling period $T$, according to the controlled plant requirements. Then, this controller is designed according to the closed-loop requirements for the ideal loop ${ }^{6}$ (depicted in Figure $1)$. It can be any controller, depending on the requirements. As a result, an output/reference transfer function $M(z)$ will be obtained:

$$
M_{c}(z)=M(z) z^{-d} ; \quad M(z)=\frac{G(z) K(z)}{1+G(z) K G(z)}
$$

Note that this set-point tracking design is independent of both, the plant delay, $d$, and the filter parameter, $\lambda$.

If necessary, the reference changes could be filtered or smoothed by means of the filter $K_{f}$

$$
K_{f}(z)=\bar{M}^{-1}(z) \frac{(1-\alpha)^{n}}{(z-\alpha)^{n}}
$$

where $\bar{M}$ is usually taken as the minimum-phase components of $M$, and $\alpha$ is the only tuning parameter in this filter.

(2) Undelayed output prediction: The undelayed output prediction $\bar{y}(8)$ (Figure 2 ) is computed by (13) and (14). It is independent of $\lambda$, although this parameter appears in both filters. Its relevance is shown up when the disturbance response or the noise effect are considered.

Note that $\lambda$, if it is convenient, can be used in the last step to reach a tradeoff between performance and robust stability. Otherwise it can be set equal to zero.

(3) Tradeoff between performance and robust stability: The tuning of $\lambda$ could be done in an analytical way according to (26) and (27), or as a rule of thumb: the larger the parameter $\lambda$ the more robust the system is but a decrease in the load disturbance rejection results.

\footnotetext{
${ }^{6}$ The tuning of this controller can be done either in the discrete or continuous time framework, to be implemented in discrete time, and it is design for the delay-free plant model.
} 
In order to improve the robustness, the predictor error filter $F_{k}(z)$ can be also included. As both indices (30) and (23) depend on the $\lambda$-parameter and the plant model but not on the designed controller, for a given plant model it is possible to provide a graphical tuning procedure to find a value of $\lambda$ to have a given tradeoff between the IAE performance and the robust stability.

\section{Illustrative Examples}

In this section the proposed GP-based control schema is applied to several processes recently used in the literature to illustrate the design procedure as well as the variety of situations that can be considered. Similar to the SP [30], the controller parameters on the proposed scheme can be tuned by following any general purpose control design tuning rules for delay-free plants, [6], or by means of a specific method for DTC in the delay free sensitivity functions [33]. Nevertheless, the predictor should take into account the actual plant delay and thus, affecting the global performances.

The results are compared with two schemes conceived to improve the performance of integrating [3] and unstable time-delay systems [19]. For a fair comparison, the proposed controller is tuned to provide similar disturbance performance, then robustness is evaluated for each scheme. Performance and robustness are compared using standard metrics.

To evaluate the control performance, the integrated absolute error (IAE) and the total variation (TV) of the manipulated input $u(t), T V=\Sigma_{i=1}^{i=\infty}\left|u_{i+1}-u_{i}\right|$, are computed. Both indices should be as small as possible [28]. To evaluate the robustness standard robustness metrics such as Gain margin $\left(M_{g}\right)$ and Phase margin $\left(\phi_{m}\right)$ are used. Some times, these figures cannot capture the idea of stability margin [6]. An alternative measure of the stability margin is the delay margin (DM), that is, the maximum extra time delay admitted before the controlled plant becomes unstable, [2], which is also considered as a robustness measurement.

Keeping in mind the waterbed effect, for integrating and unstable time-delay systems the most critical point is the tradeoff between disturbance rejection and robustness. The set-point tracking can be always modified by an ad-hoc set-point filter. Note that, for unstable systems, robustness cannot be increased arbitrarily. This is an expected result because certain feedback action is needed to maintain stability, thus, the detuning of the controller has a limit [26].

To illustrate different properties, the following systems will be considered:

(1) An integrating process with dead-time [3], to show a detailed application of the design procedure.
(2) A double integrator process with a dead-time, also treated in [3].

(3) An unstable system taken from [19].

(4) The two previous cases, 1 and 3, initially assumed with the same delays as reported in the referred papers, but now with a very long delay.

Example 1: Let us consider an integrating process with dead-time [3]:

$$
P_{p}(s)=\frac{e^{-4 s}}{s(s+1)}
$$

Following the design procedure, let us consider the scheme in Figure 1. The tuning of the controller can be done to fulfill some requirements, for example, a $0 \%$ overshoot and recovery time of 60 s for step load disturbance. Then, the PID controller for the delay-free plant could be such as:

$$
K(s)=0.21 \frac{(s+1)(s+1 / 20)}{s(0.1 s+1)}
$$

A suitable sampling time, such as $T=0.2 \mathrm{~s}$, is considered, although it could be larger. The discrete time controller $K(z)$ is obtained by discretizing $K(s)$, and the discrete process model is:

$G(z)=\frac{0.018731(z+0.9355)}{(z-1)(z-0.8187)}$

Following the design procedure (second step), with $\lambda=$ 0 :

$$
\begin{aligned}
\Gamma(z) & =\frac{0.018731(z+0.9355)}{z} \\
\tilde{G}(z) & =\frac{z}{(z-1)(z-0.8187)}
\end{aligned}
$$

Thence, the filters $F_{1}(z)$ and $F_{2}(z)$ are computed from (32) and (33) by (13) and (14) respectively with $d=$ $L / T=20$. The obtained results, assuming $F_{k}(z)=1$, compared with the ideal closed-loop delay-free system (see Figure 1), are shown in Figure 6.

In order to improve the robust tuning of the overall closed-loop system the parameter $\lambda$ should be adjusted (third step). In this example, and in order to compare with the proposal in [3], the parameter $\lambda$ is computed from (30) to obtain in both a similar IAE (see Table 1), being taken as $\lambda=0.84$.

$$
\begin{aligned}
\Gamma(z) & =\frac{0.0048374(z+0.9672)}{(z-0.84)} \\
\tilde{G} & =\frac{(z-0.84)}{(z-1)(z-0.9048)}
\end{aligned}
$$




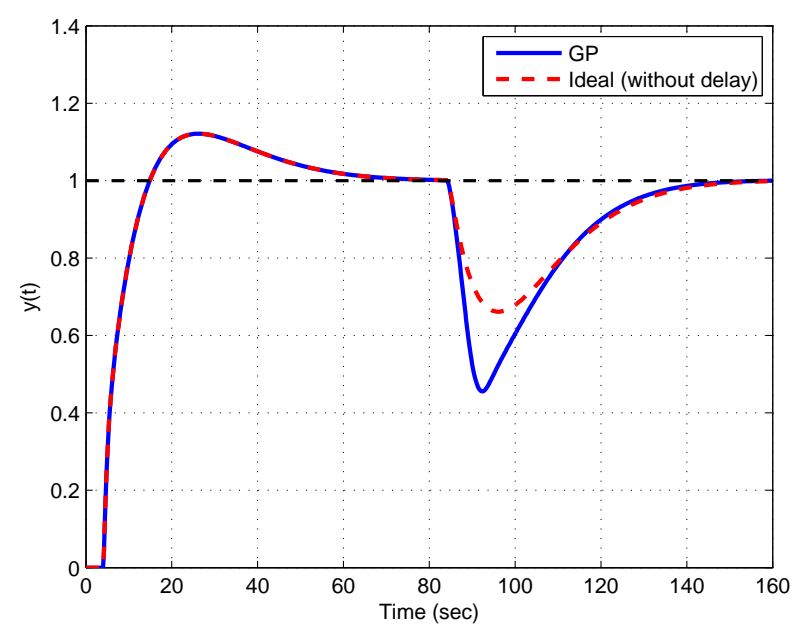

Figure 6. Nominal output response compared with the ideal closed-loop system free of delay for a unit step change in the reference and a step load disturbance of -0.1 at $t=80$ s. (Example 1).

Table 1

Performance \& Robustness (nominal case of Example 1)

\begin{tabular}{c|c|c||c|c|c}
\hline \hline Load disturbance & $I A E$ & $T V$ & $M_{g}$ & $\phi_{m}$ & $D M$ \\
\hline$[3]$ & 11.78 & 0.20 & 1.65 & 50.50 & 4.35 \\
\hline GP & 11.72 & 0.15 & 1.94 & 44.16 & 4.38 \\
\hline
\end{tabular}

$I A E$ and $T V$ are computed for load disturbance case $(t>80$ s in Figure 7$)$.

Thence, the filters $F_{1}(z)$ and $F_{2}(z)$ are computed from (34) and (35) with $d=20$, the predictor error filter being:

$$
F_{k}(z)=\frac{(1-0.84)}{(z-0.84)}
$$

In [3], the following parallel PID-controller is designed based on the delayed plant model:

$$
P I D(s)=k c\left(1+\frac{T_{d} s}{T_{f} s+1}+\frac{1}{T_{i} s}\right)
$$

where $k_{c}=0.19, T_{i}=22.37, T_{d}=2.79$ and $T_{f}=0.279$, are the result of an optimization process where the delayed plant model is used.

For the nominal case, the results obtained, compared with those in [3] are shown in Figure 7, 8 and Table 1, where the different metrics for performance and robustness are collected.

Similar to [3], the robust performances of the proposed scheme is studied by assuming a $20 \%$ change in the steady state gain or the plant delay. The results obtained, compared with those in [3], are shown in Figure
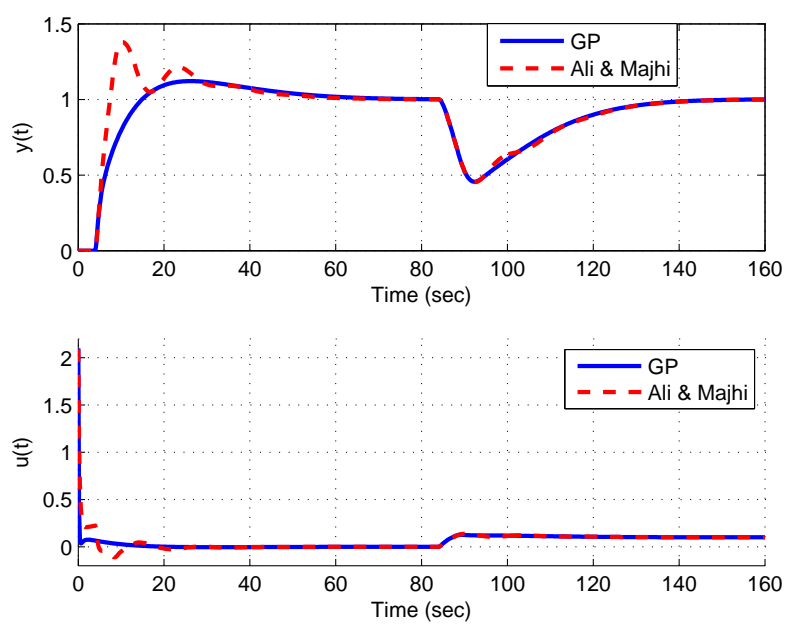

Figure 7. Nominal system responses for a unit step change in the reference and a -0.1 step load disturbance at $t=80 \mathrm{~s}$ (Example 1).

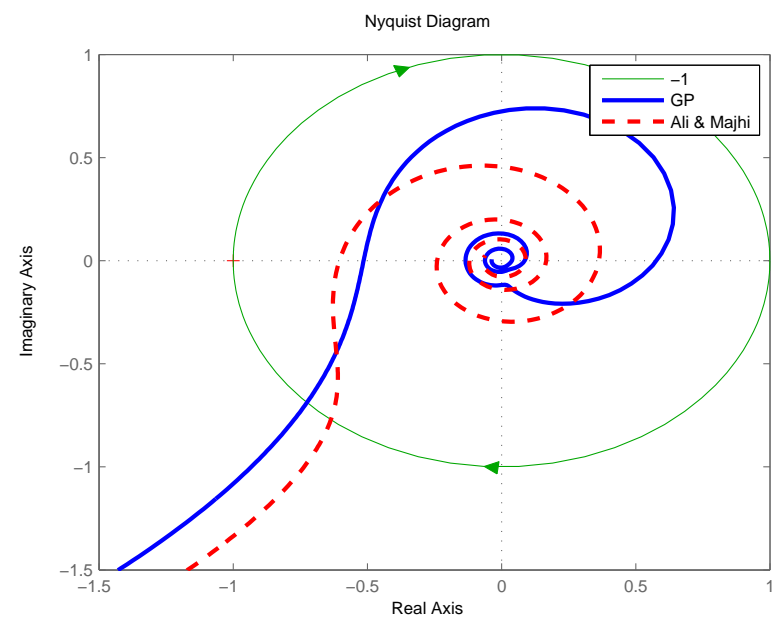

Figure 8. Nyquist plots for Example 1.

Table 2

\begin{tabular}{|c|c|c|c|c|}
\hline & \multicolumn{2}{|c|}{$+20 \%$ gain } & \multicolumn{2}{|c|}{$+20 \%$ time delay } \\
\hline & $I A E$ & $T V$ & $I A E$ & $T V$ \\
\hline [3] & 11.76 & 0.34 & 11.77 & 0.26 \\
\hline GP & 11.69 & 0.21 & 11.70 & 0.19 \\
\hline
\end{tabular}

Performance \& Robustness (perturbed cases Example 1)

$\overline{I A E}$ and $T V$ are computed as before, for load disturbance, with a $20 \%$ change in the steady state gain and the plant delay.

9 and Figure 10 and Table 2. In both cases, the IAE is similar for both schemes but the TV is better in the proposed scheme.

If the modeling errors are increased in both, the delay and gain, the difference could be greater. For a $40 \%$ 

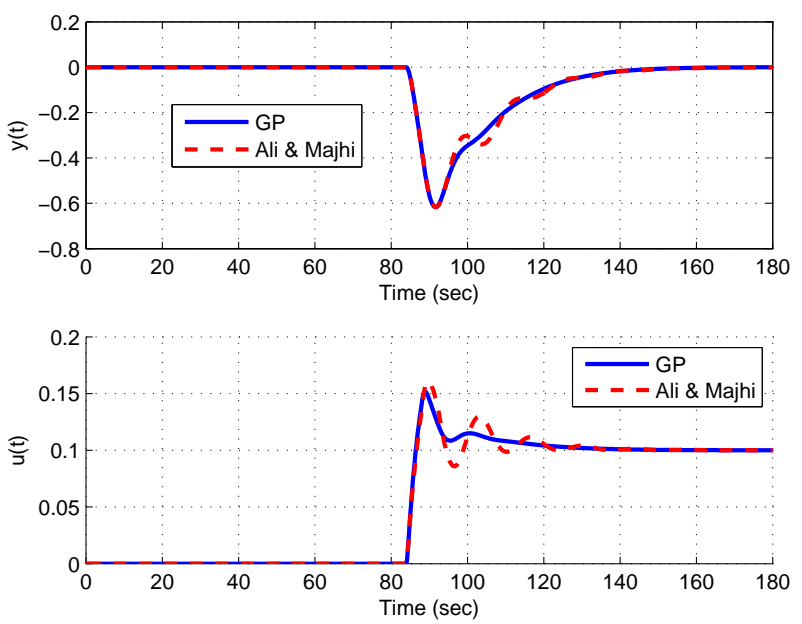

Figure 9. Responses for a change of $20 \%$ in process gain (Example 1).
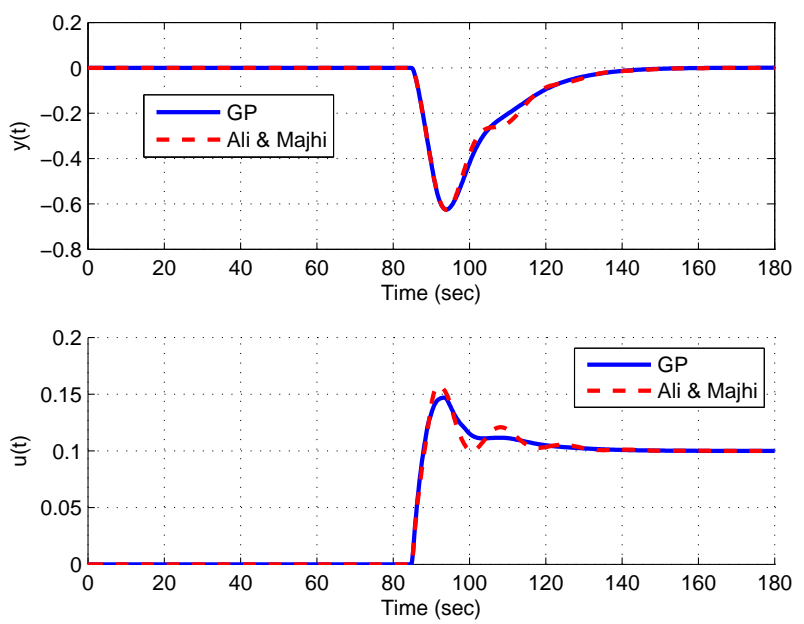

Figure 10. Responses for a change of $20 \%$ in process time delay (Example 1).

modeling errors, the proposal of [3] becomes unstable whereas the GP remains stable (see Nyquist plot in Figure 11), but it is fair to note that the differences are not so large and both designs are acceptable for reduced model mismatching and low time delays.

Finally, let us consider the effect of a white noise in the measurement device, with a power spectrum of 0.02 . For the nominal case, the results are shown in Figure 12.

For this example it is worth to conclude that, if both schemes are tuned to have the same IAE in the nominal case, the phase margin is slightly higher in the scheme proposed by Ali \& Maihi [3], but the gain margin and the Nyquist plot distance to the $(-1,0)$ point $\left(M_{s}\right)$ are lower, the DM being similar. For gain or delay uncertainties the computed IAE is similar for both schemes but

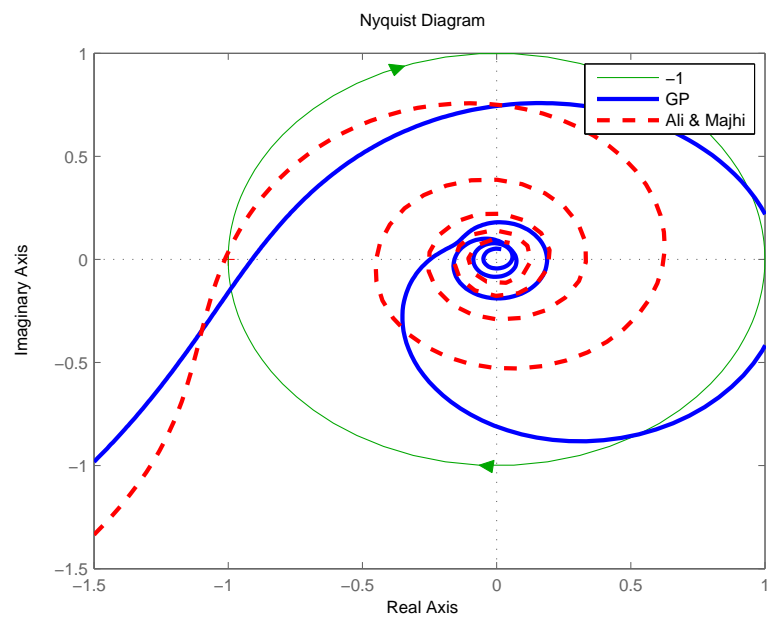

Figure 11. Nyquist plots, for the perturbed case when time delay and gain are increased by $40 \%$ (Example 1).
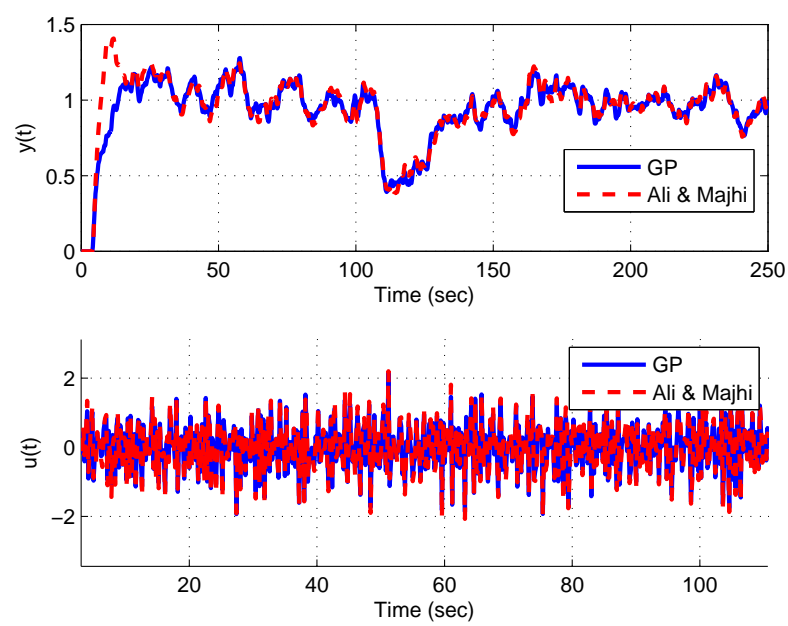

Figure 12. Nominal system responses for white-noise measurement (Example 1).

the TV is better in the GP. For simultaneous uncertainties (in both delay and gain), the proposed scheme shows better robustness again uncertainties and load disturbance rejection.

Example 2: Now let us consider the same integrating process but with a much higher dead-time:

$P_{p}(s)=\frac{e^{-12 s}}{s(s+1)}$

The authors in [3] announce major difficulties in the controller design for dead-time greater than 10, and "for delay dominated plants, the Smith predictor should be used for integrating plant models with large time de- 


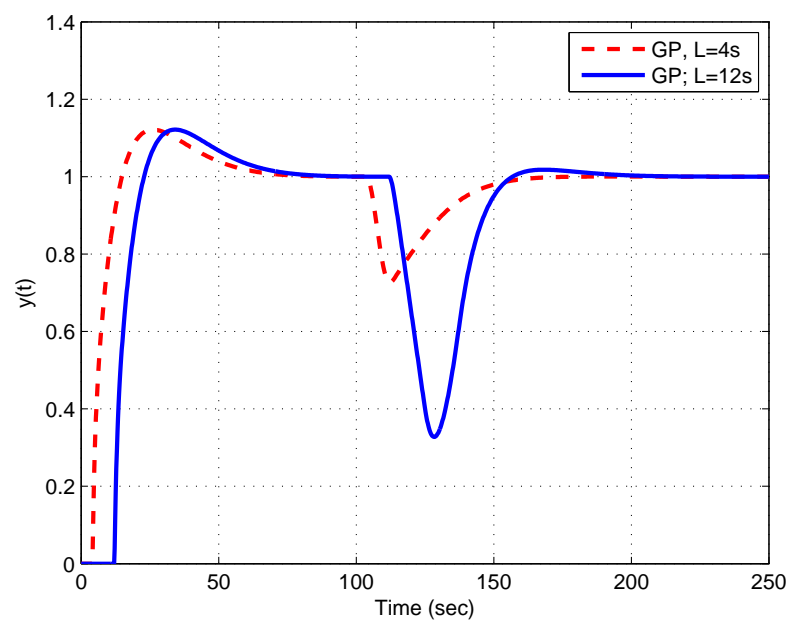

Figure 13. Nominal system responses for a -0.05 step disturbance at $t=100$ s (Example 2).

lay" 7 .

Following the proposed methodology, the controller $K(z)$ and the predictor error filter $F_{k}(z)$ are initially the same that those computed in the previous case. The filters $F_{1}(z)$ and $F_{2}(z)$ are computed from (34) and (35), with the same sampling period $T=0.2 \mathrm{~s}$, but with $d=L / T=60$. Then, the comparative results as a function of the delay are shown in Figure 13. The worsening of the robustness against model uncertainties is shown in Figure 14.

Note that as expected by the nature of the integrative systems, the robustness and the load disturbance rejection are degraded as far as the time delay increases. If necessary, a robustness improvement can be done by the $\lambda$-parameter, but at the expense of decreasing the load disturbance rejection. Also, the initially designed robust control $K(z)$ can be redesigned.

Example 3: Let us now consider a double integrator process with a dead-time, also treated in [3]:

$P_{p}(s)=\frac{e^{-s}}{s^{2}}$

Following the design procedure, the tuning of the controller can be done to fulfill some requirements for a step load disturbance, for example:

$$
K(s)=0.20 \frac{(2.5 s+1)(s+1 / 5)}{s(0.25 s+1)}
$$

\footnotetext{
7 Quoted from [3]
}
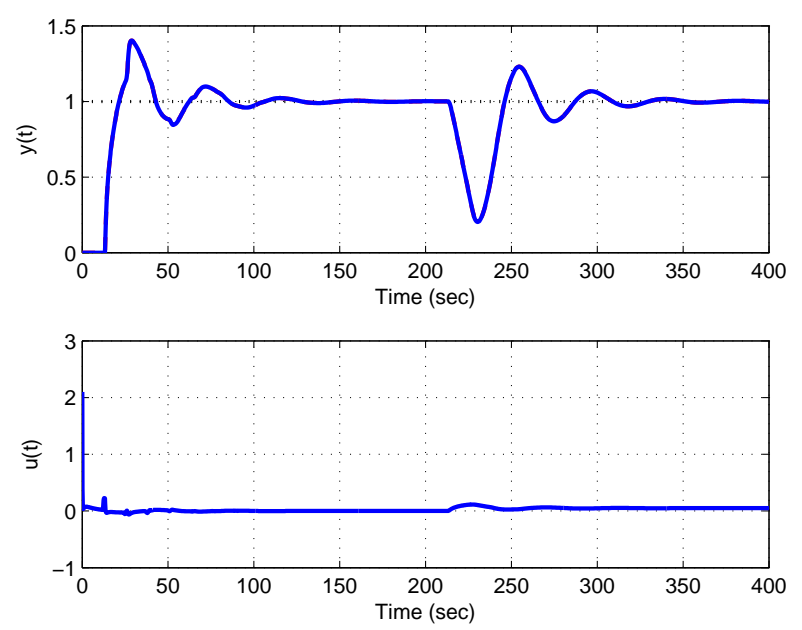

Figure 14. Perturbed system responses when the delay and gain are increased by $10 \%$ for a -0.05 step disturbance at $t=100$ s (Example 2).

The following set-point filter could be included if the overshoot should be avoided:

$$
K_{f}(s)=\frac{M(s)^{-1}}{(3 s+1)^{2}}
$$

The discrete time controllers $K(z)$ and $K_{f}(z)$ are obtained by discretizing the previous $K(s)$ and $K_{f}(s)$, where $T=0.2 \mathrm{~s}$. The discrete process model is:

$G(z)=\frac{0.02(z+1)}{(z-1)^{2}}$

Note that a zero appears at $z=-1$. Then, following the design procedure, the $\lambda$ parameter should be adjusted. In this example, and in order to compare with the proposal in [3], $\lambda=0.953$ is selected to obtain the same IAE for the nominal case there proposed (see Table 3 ).

$$
\begin{aligned}
\Gamma(z) & =\frac{0.02(z+1)}{(z-0.953)} \\
\tilde{G} & =\frac{(z-0.953)}{(z-1)^{2}}
\end{aligned}
$$

Thence, the filters $F_{1}(z)$ and $F_{2}(z)$ are computed from (36) and (37) by (13) and (14) respectively.

As before, in [3], the following PID is proposed:

$$
P I D(s)=k c\left(1+\frac{T_{d} s}{T_{f} s+1}+\frac{1}{T_{i} s}\right)
$$

where a set-point filter is also included in order to avoid 
Table 3

Nominal Performance \& Robustness (Example 3)

\begin{tabular}{c|c|c||c|c|c}
\hline \hline Load disturbances & $I A E$ & $T V$ & $M_{g}$ & $\phi_{m}$ & $D M$ \\
\hline$[3]$ & 25.17 & 0.46 & 1.82 & 15.21 & 0.47 \\
\hline GP & 24.73 & 0.29 & 1.95 & 19.47 & 0.70 \\
\hline
\end{tabular}
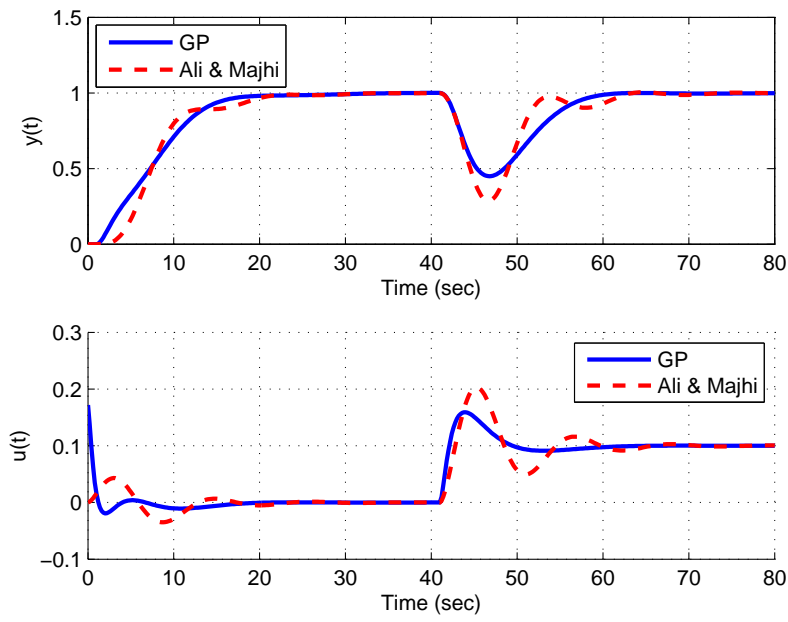

Figure 15. Nominal system responses for a -0.1 step disturbance at $t=100$ s (Example 3 ).

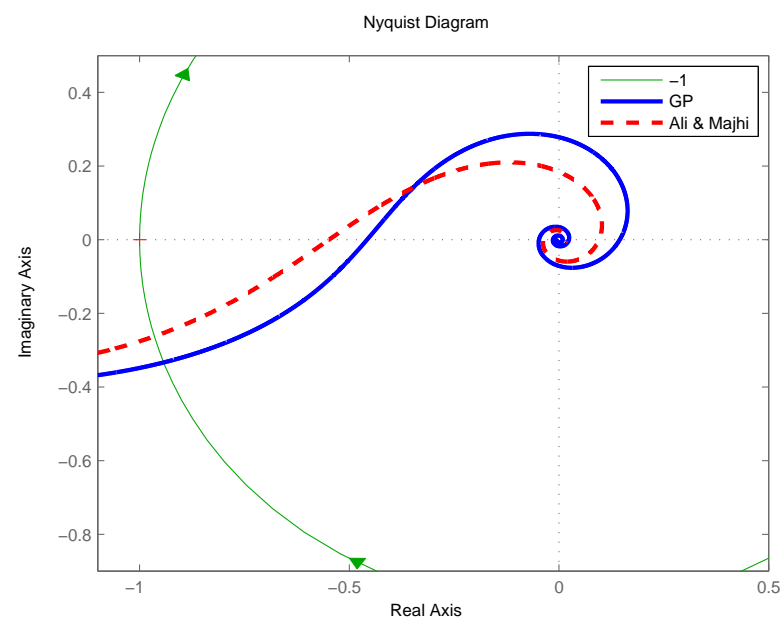

Figure 16. Nyquist plots for Example 3.

the overshoot:

$$
F_{s p}(s)=\frac{1}{T_{i} T_{d} s^{2}+T_{i} s+1}
$$

with $k_{c}=0.17, T_{i}=8.51, T_{d}=2.87$ and $T_{f}=0.287$.

The results obtained, compared with those in [3] are shown in Figure 15 and Table 3 (see also the Nyquist plots in Figure 16).
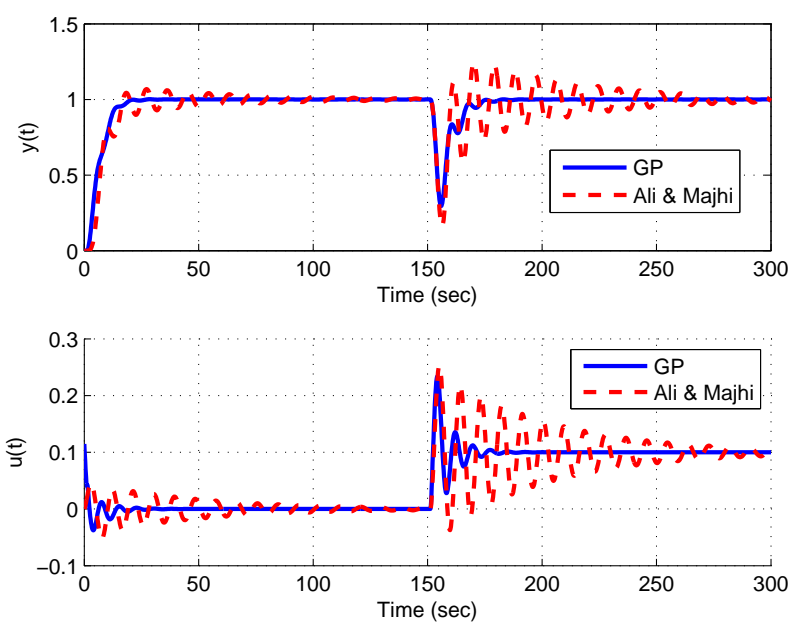

Figure 17. Closed loop responses for the perturbed case when the delay and gain are increased by $25 \%$ (Example 3 ).

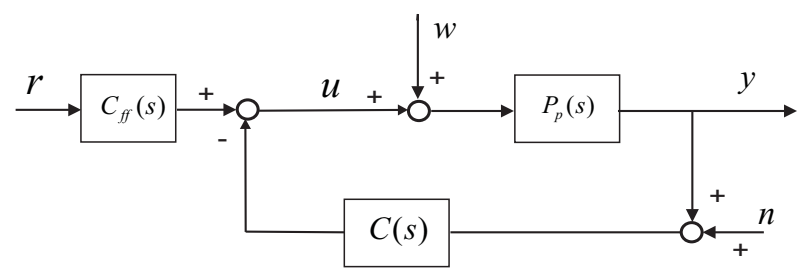

Figure 18. MSP-PID control structure proposed in [19].

If the modeling errors are increased by $25 \%$ in both, the delay and gain, the results are shown in Figure 17.

Again, if the modeling errors are increased by $50 \%$ the proposal of [3] becomes unstable whereas the GP remains stable.

Thus, for a double integrator process, it is possible to conclude that, if both schemes are tuned to have a similar IAE for regulatory proposes in the nominal case, the proposed scheme shows a slightly improvement in the performance and robustness again uncertainties. Note that also the DM is higher in the GP.

Example 4: Let us consider an unstable processes with a dead-time [19]:

$P_{p}(s)=\frac{2}{(10 s-1)(2 s+1)} e^{-5 s}$

In the scheme proposed in [19], the so called MSP-PID, the following controllers are proposed (see Figure 18):

$$
C(s)=\gamma \frac{k_{d} s^{2}+k s+k_{i}}{s\left(T_{f} s+1\right)} F_{c}(s)
$$


for the output feedback, and

$$
C_{f f}(s)=\gamma \frac{b k s+k_{i}}{s} F_{c}(s)
$$

for the set-point tracking, and

$$
F_{c}(s)=\frac{\left(a_{2} s^{2}+a_{1} s+1\right)}{\left(a_{2} s^{2}+a_{1} s+1+\eta\right)}
$$

where $\gamma=1, k=4.1452, k_{d}=6.7044, k_{i}=0.1960$, $T_{f}=0.2871, b=0, \eta=2.864$ and $a_{1}=\tau / 2$, and $a_{2}=\tau^{2} / 12$ with $\tau=7$ are the result of an optimization process where the delayed plant model is used. Following the tuning procedure here proposed, the main controller and set-point filter are tuned to fulfill the closed-loop stability. Assume, for example, the PID controller ${ }^{8}$ :

$$
K(s)=2.9 \frac{(2 s+1)(s+1 / 10)}{s(0.1 s+1)}
$$

The set-point filter, can be taken as:

$$
K_{f}(s)=M^{-1}(s) \frac{1}{(\alpha s+1)^{2}}
$$

with $\alpha=5$;

The proposed scheme (Figure 2) is implemented with a sampling time of $T=0.1 \mathrm{~s}$. The parameter $\lambda$ is adjusted to obtain a IAE slightly lower than the one in the MSPPID, that is with $\lambda=0.98$ the IAE is 26.54 (see Table 4), then:

$$
\begin{aligned}
\Gamma(z) & =\frac{0.00049342(z+0.9868)}{(z-0.98)} \\
\tilde{G} & =\frac{(z-0.98)}{z-1.01)(z-0.9512)}
\end{aligned}
$$

Thence, the filters $F_{1}(z)$ and $F_{2}(z)$ are computed from (38) and (39) with $d=50$, being $F_{k}(z)=\frac{(1-0.98)}{z-0.0 .98}$.

The obtained results, compared with those in [19], are shown in Figure 19 and Table 4 (see also Nyquist plots in Figure 21). In Figure 20 the same simulation but including a unit step change in the set-point is considered.

In order to evaluate the robust performances of the proposed scheme, a variation of $-18 \%$ in all process parameters is assumed, as done in [19]. The obtained results, are shown in Figure 22.

\footnotetext{
8 This controller should be designed according to the disturbance rejection requirements, in the general case.
}

Table 4

Performance \& robustness (Example 4)

\begin{tabular}{c|c|c||c|c|c}
\hline \hline Load disturbances & $I A E$ & $\mathrm{TV}$ & $M_{g}$ & $\phi_{m}$ & $D M$ \\
\hline$[19]$ & 28.57 & 0.53 & 1.36 & 15.22 & 1.33 \\
\hline $\mathrm{GP}$ & 26.54 & 0.42 & 1.40 & 17.11 & 1.51 \\
\hline
\end{tabular}
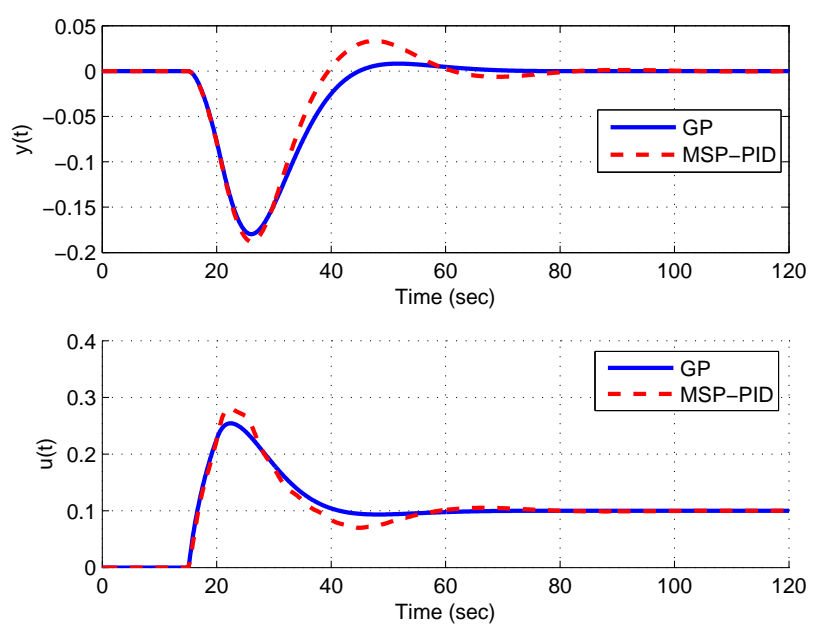

Figure 19. Nominal system responses for a -0.1 step disturbance at $t=10$ s (Example 4 ).
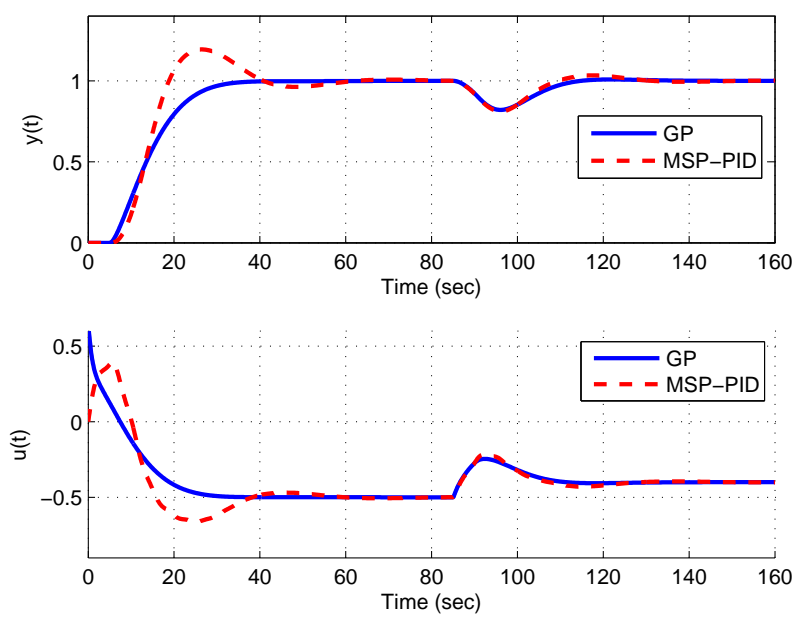

Figure 20. Nominal system responses for an unit step in the set-point and a -0.1 step disturbance at $t=80$ s (Example $4)$

But, if the parameter variations in the process are $+25 \%$ in the time delay and $-25 \%$ in the other parameters, that is, for

$P_{p}(s)=\frac{0.13333 e^{-6.3 s}}{(s+0.6667)(s-0.1333)}$

the results obtained, compared with those in [19], are 


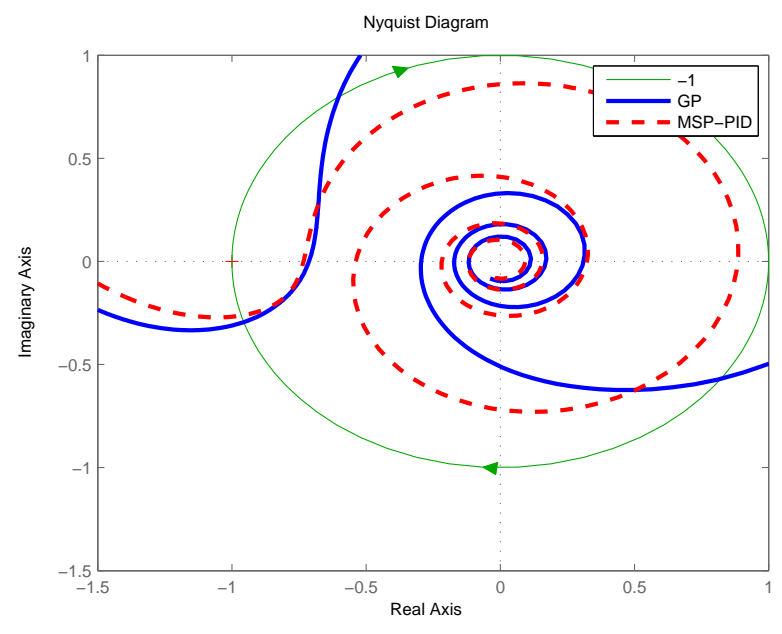

Figure 21. Nyquist plots for Example 4.
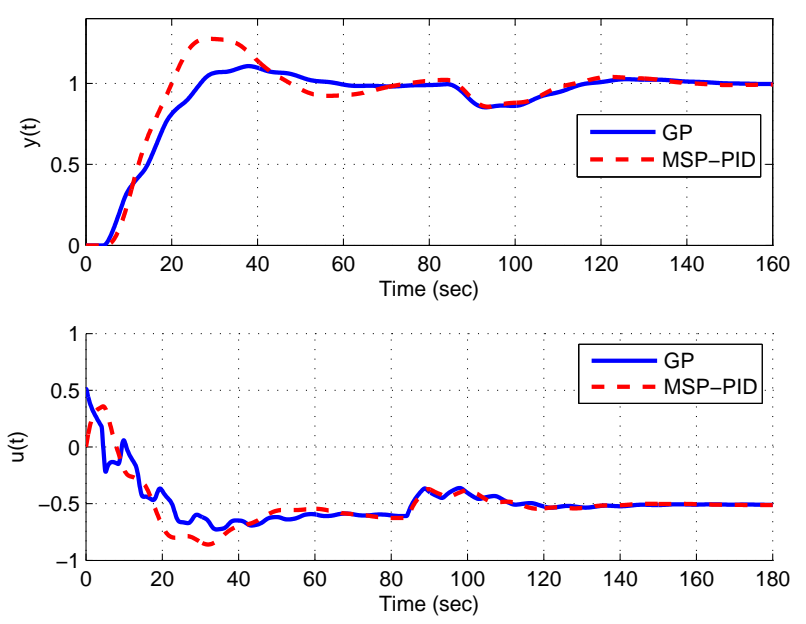

Figure 22. Perturbed system responses; all process parameters are changed by $-18 \%$ (Example 4 ).

shown in Figure 23. Note that the control proposed in [19] becomes unstable whereas the GP is stable.

Example 5: Let us consider the same unstable processes but with a longer dead-time:

$P_{p}(s)=\frac{2}{(10 s-1)(2 s+1)} e^{-12 s}$

The proposed scheme has been implemented with the filters $F_{1}(z)$ and $F_{2}(z)$ computed from (38) and (39), but with $d=L / T=120$. Being the controller $K(z)$ and the predictor error filter $F_{k}(z)$ the same that in the previous example. Then, the results in Figure 24 and 25 are obtained.

As pointed out in Example 2, the proposed controller

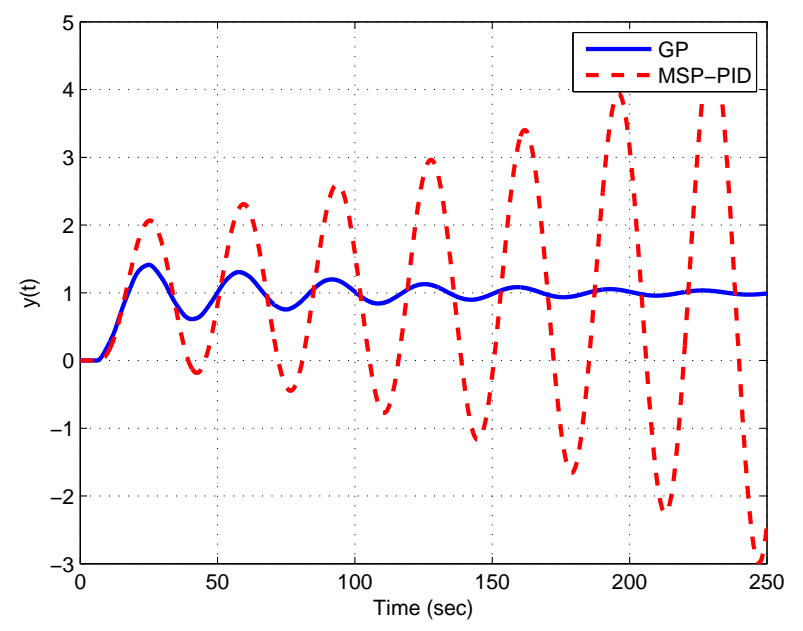

Figure 23. Closed loop responses for the perturbed case when the time delay is increased by $25 \%$ and other parameters are decreased by $-25 \%$ (Example 4 )
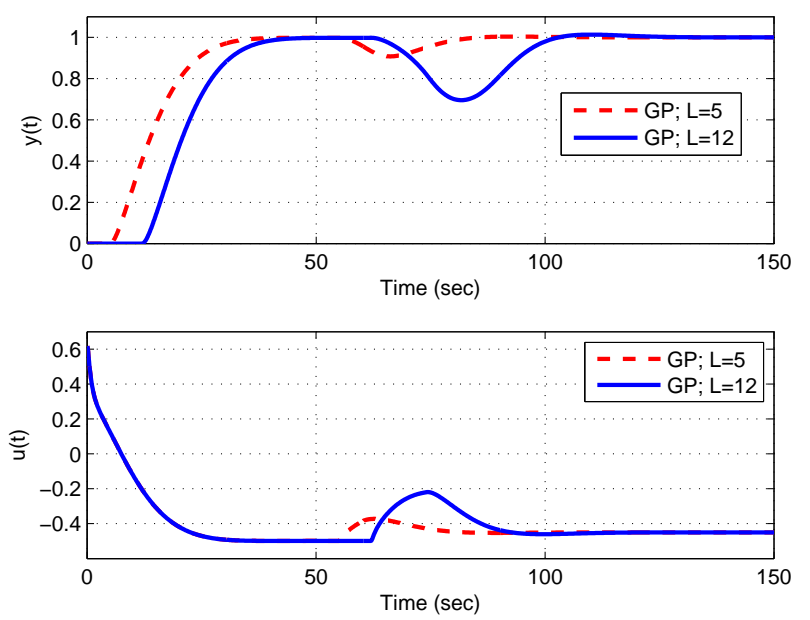

Figure 24. Nominal system responses for a -0.05 step load disturbance at $t=100$ s (Example 5).

design methodology is independent on the delay but, as expected by the nature of the unstable plant, the robustness index and the load disturbance rejection are degraded as far as the time delay increases. If necessary, a robustness improvement can be done by the $\lambda$ parameter, but at the expense of decreasing the load disturbance rejection.

\section{Conclusions}

The GP has been shown to be a convenient alternative to the classical SP, allowing the delay-free control design for stable, integrating or unstable processes with large time delay. Based on this new predictor, where a new tuning parameter has been included, a control structure 


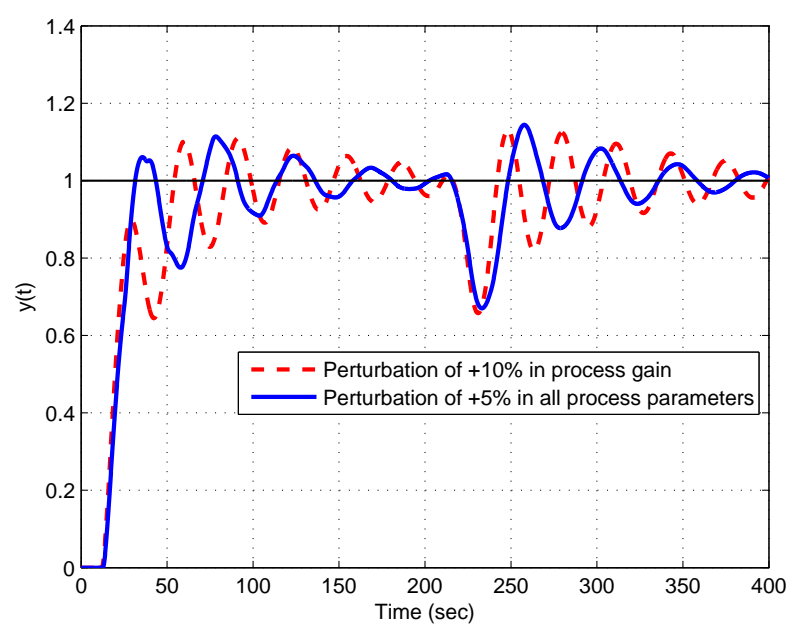

Figure 25. Perturbed system responses for a -0.05 step load disturbance at $t=200$ s (Example 5 ).

has been proposed. It can be seen that the $\lambda$-parameter can improve the robustness, the larger the $\lambda$-parameter the lower the disturbance performance is obtained but an increment in the robustness results. This new parameter can also be introduced in the prediction error filter to get an intuitive tradeoff between performance and robust stability. The main advantages of the proposed structure is the easy tuning and industrial implementation as well as the intuitive effect of the control parameters. Similarly to the original SP, any suitable control structure and design for the free-delay process can be applied, independently on the proposed GP.

The proposed control scheme has been applied and the results compared to those recently published by using alternative approaches, where excellent results were claimed. In the reported examples, disturbance rejection and robustness against model uncertainties and measurement noise have been considered. Our proposal, as described in the previous sections, is general, applicable to any kind of plants with long time delays and it can be easily designed and implemented, leading to better results.

It is worth to remark that the main controllers are designed independently of the delay. The same controllers are applied for the same system if the time delay is changed, and the reference responses are the same in all cases, as can be seen in Figures 6, 13 or 24, where the delay effect on disturbance rejection is also shown. The delay is taken into account in designing the predictor and its negative effects can be balanced by means of the tuning parameter $\lambda$.

\section{Acknowledgments}

This work has been partially granted by the Spanish Ministry of Education research Grants DPI2011-28507C02-01 and PAID-06-12. The authors are also grateful to the Associate Editor and anonymous reviewers for their valuable feedback and comments.

\section{References}

[1] P. Albertos and P. García. Robust control design for long time-delay systems. Journal of Process Control, 19(10):1640-1648, 2009.

[2] Pedro Albertos. Phase-conditionally stable systems. Systems \& control letters, 55(10):803-808, 2006.

[3] Ahmad Ali and Somanath Majhi. PID controller tuning for integrating processes. ISA transactions, 49(1):70-78, 2010.

[4] K. J. Åström. Model uncertainty and feedback. In P. Albertos and A. Sala Piqueras, editors, Iterative Identification and Control. Springer Verlag. (2002).

[5] K. J. Åström, C.C. Hang, and B.C. Lim. A new smith predictor for controlling a process with an integrator and long dead time. IEEE Transactions on Automatic Control 39 (1994) 343-345.

[6] K.J. Åström and T. Hägglund. Advanced PID Control. ISA - The Instrumentation, Systems, and Automation Society, Research Triangle Park, NC 27709, 2005.

[7] K.J. Åström and B. Wittenmark. Computer Controlled Systems: Theory and Design, Third Edition. Englewood Cliffs, NJ: Pretince-Hall, 1997.

[8] P. García, P. Albertos, and T. Hägglund. Control of unstable non-minimum-phase delayed systems. Journal of Process Control, 16:1099-1111, 2006.

[9] P. García and P. Albertos. A new dead-time compensator to control stable and integrating processes with long dead-time. Automatica, 44(4):1062-1071, 2008.

[10] P. García, T. Santos, J. E. Normey-Rico, and P. Albertos. Smith predictor-based control schemes for dead-time unstable cascade processes. Ind. Eng. Chem. Res. 49 (2010) 11471-11481.

[11] J.L. Guzmán, P. García, T. Hägglund, S. Dormido, P. Albertos, and M. Berenguel. Interactive tool for analysis of time-delay systems with deadtime compensators. Control Engineering Practice, $16(7): 824-835,2008$.

[12] T. Hägglund. An industrial dead-time compensating PI controller. Control Eng. Practice 4 (1996) 749-756.

[13] T. Hägglund. A predictive PI controller for process with long dead-times. IEEE Contr. Syst. Mag. 12 (1) (1992) 57-60.

[14] A. Ingimundarson and T. Hägglund. Robust tuning procedures of dead-time compensating con- 
trollers. Control Engineering Practice, 9(11):11951208, 2001.

[15] T. Liu, W. Zhang, and D. Gu. Analytical design of two-degree-of-freedom control scheme for open-loop unstable processes with delay. Journal of Process Control 15 (2005) 559-572.

[16] X. Lu, Y.-S. Yang, Q.-G. Wang, and W.-X. Zheng. A double two-degree-of-freedom control scheme for improved control of unstable delay processes. Journal of Process Control 15 (2005) 605-614.

[17] S. Majhi and D.P. Atherton. Obtaining controller parameters for a new smith predictor using autotuning. Automatica 36 (2000) 1651-1658.

[18] M.R. Mataušek and Micié A.D. On the modified smith predictor for controlling a process with an integrator and long dead-time. IEEE Transactions on Automatic Control 44 (1999) 1603-1606.

[19] MR Mataušek and AI Ribić. Control of stable, integrating and unstable processes by the modified smith predictor. Journal of Process Control 22 (2012) 338-343.

[20] W. Michiels and S.I. Niculescu. On the delay sensitivity of smith predictors. International Journal of Systems Science 34 (2003) 543-552.

[21] J.E. Normey-Rico, C. Bordons, and E.F. Camacho. Improving the robustness of dead-time compensating pi controllers. Control Eng. Practice 5 (1997) 801-810.

[22] J.E. Normey-Rico and Eduardo. F. Camacho. Dead-time compensators: A survey. Control Engineering Practice, 16(4):407-428, 2008.

[23] J.E. Normey-Rico and E.F. Camacho. Control of dead-time processes. Spinger, Berlin, 2007.

[24] J.E. Normey-Rico and E.F. Camacho. Unified approach for robust dead-time compensator design. Journal of Process Control, 19 (2009) 38-47.

[25] J.E. Normey-Rico and E.F. Camacho. A unified approach to design dead-time compensators for stable and integrative process with dead-time. IEEE Transactions on Automatic Control 47 (2002) 299-305.

[26] J.E. Normey-Rico and E.F. Camacho. Unified approach for robust dead-time compensator design. Journal of Process Control, 19(1):38-47, 2009.

[27] A. Seshagiri Rao and M. Chidambaram. PI/PID controllers design for integrating and unstable systems (chapter 3), pages 75-111. In R. Vilanova, A. Visioli (eds.), PID control in the third millennium, advances in industrial control, 2012.

[28] S. Skogestad. Simple analytic rules for model reduction and PID controller tuning. Journal of process control, 13(4):291-309, 2003.

[29] S. Skogestad and I. Postlethwaite. Multivariable feedback control analysis and design. Published by Wiley, May 1996.

[30] O.J.M. Smith. Closer control of loops with dead time. Chemical Engineering Progress 53 (1959) 217-219.

[31] W. Tan, H.J. Marquez, and T. Chen. Imc desing for unstable processes with time delays. Journal of Process Control 13 (2003) 203-213.

[32] Y.-C. Tian and F. Gao. Double-control scheme for control of processes with dominant delay. IEE Proc.-Control Theory and Appl., Vol. 145, pp. 479-484, (1998).

[33] A. Visioli and Q. Zhong. Control of integral processes with dead time. Springer, 2010.

[34] Q.-G. Wang, H.-Q. Zhou, Y. Zhang, and Y. Zhang. A comparative study on control of unstable processes with time delay. 5th Asian Control Conference, Melbourne , Australia, pages 20062014, July 2004.

[35] Q.-C. Zhong and J. Normey-Rico. Control of integral processes with dead-time. part 1: Disturbance observer-based 2 dof control scheme. Control Theory and Applications, IEEE Proceedings 149 (4) (2002) 285-290. 